\title{
Síndrome epiléptico ligado al cromosoma $X$ por mutación de la protocaderina 19 (OMIM 300088) asociado con leucoencefalopatía y tractopatía posterior reversible
}

\author{
Blair Ortiz¹, Yesyka Jaramillo', Christian Rojas² \\ ${ }^{1}$ Grupo de Neurología Infantil, Universidad de Antioquia, Medellín, Colombia \\ ${ }^{2}$ Facultad de Salud, Universidad del Valle, Cali, Colombia \\ La epilepsia con retardo mental ligado al cromosoma X por mutación del gen PCDH19, es una condición \\ que solo se presenta en las mujeres. El cuadro clínico suele verse complicado con retardo global del \\ desarrollo y epilepsia. En la edad adulta puede manifestarse con discapacidad intelectual y hasta 20 \\ $\%$ de las mujeres afectadas no presentan convulsiones ni retardo intelectual. Se presenta el caso de \\ una niña con epilepsia, retardo del desarrollo y conversión autista, asociados con leucoencefalopatía y \\ tractopatía posterior reversible por mutación del PCDH19 (c.142G>T/ p.Glu48X).
}

Palabras clave: epilepsia; convulsiones febriles; discapacidad intelectual; leucoencefalopatías; encefalopatías.

doi: https://doi.org/10.7705/biomedica.v38i4.3900

\section{X-linked epileptic syndrome by protocadherin 19 mutation associated with leukoencephalopathy and posterior reversible tractopathy}

Epilepsy and mental retardation produced by mutations in gene $P C D H 19$ (protocadherin 19) is an $X$-linked syndrome restricted to females. It starts with global and speech developmental delay and epilepsy; intellectual disability may continue in adults. At least in $20 \%$ of cases, there are no seizures or intellectual retardation. We report the case of a girl with epilepsy, developmental delay, and autistic conversion associated with posterior reversible leukoencephalopathy and tractopathy produced by $P C D H 19$ mutation (c.142G>T/ p.Glu48X).

Key words: Epilepsy; seizures, febrile; intellectual disability; leukoencephalopathies; brain diseases. doi: https://doi.org/10.7705/biomedica.v38i4.3900

Las protocaderinas, proteínas transmembrana de adhesión cuya estabilidad mecánica depende del calcio, constituyen un subgrupo de la superfamilia de las caderinas. Se han reconocido más de 70 genes de protocaderinas (1,2). En el sistema nervioso central están ampliamente involucradas en el proceso de neurulación anterior y en la morfogénesis.

Son numerosas las enfermedades cerebrales de etiología metabólica y genética que producen encefalopatía progresiva y pueden estar asociadas con la epilepsia. La encefalopatía ligada al cromosoma X por mutación del gen $\mathrm{PCDH} 19$ que codifica para la protocadherina 19 , se caracteriza por epilepsia resistente, trastorno de espectro autista, retardo del desarrollo, síndrome hipotónico, microcefalia y convulsiones recurrentes. Las crisis epilépticas pueden desencadenarse por diversos estímulos, como fiebre, vacunación, cirugías o infecciones adquiridas.

El PCDH19 se localiza en el cromosoma Xq22.3 y su función se expresa en el desarrollo cerebral, específicamente en la zona subventricular intermedia, subplato, capas II,IV,V y VI de la corteza cerebral, en el hipocampo y el subículo $(3,4)$. La proteína protocadherina 19 podría regular la formación de conexiones neuronales durante el desarrollo cerebral y remodelar las conexiones sinápticas durante la etapa posnatal temprana $(5,6)$.

Contribución de los autores:

Todos los autores contribuyeron a recopilar la información relevante, organizar cronológicamente el manuscrito, analizar los datos clínicos y los resultados de laboratorio, hacer la búsqueda bibliográfica ampliada y actualizar la concepción médica de la enfermedad. 


\section{Reporte del caso}

Se trata de una niña nacida de un segundo embarazo, con adaptación y antropometría adecuadas para la edad de gestación, pero con síndrome hipotónico. Su madre había tenido un aborto cuya causa no pudo establecerse. A los 10 meses de edad, aparecieron las primeras convulsiones de diferente tipo: focales, discognitivas, autonómicas y tónico-clónicas, algunas de las cuales fueron desencadenadas por la fiebre debida a una infección respiratoria aguda. La condición evolucionó a epilepsia resistente, con regresión del neurodesarrollo y del habla, agnosia visual y auditiva, y conversión autista. La evaluación neuropsicológica corroboró estos hallazgos y y se obtuvo un puntaje de 59, el cual indica moderada afectación, en la escala Bayley de desarrollo infantil.

La resonancia magnética (RM) cerebral simple practicadaalos 10 meses, mostrabaleucoencefalopatía parieto-occipital, e hiperintensidad del tracto tegmental central y de los núcleos delgado y cuneiforme (figura 1). En el electroencefalograma (EEG) se evidenció actividad epileptiforme focal témporo-parietal izquierda. Con los estudios metabólicos se descartó aminoacidopatía, aciduria orgánica, leucodistrofia metacromática y enfermedad del peroxisoma.

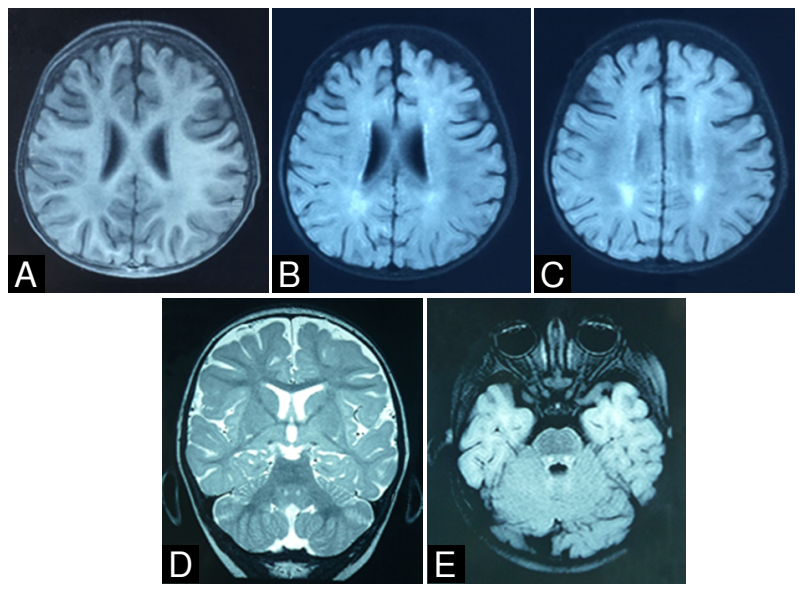

Figura 1. Resonancia magnética cerebral a los 10 meses de vida de la paciente. Se observa leucoencefalopatía parietooccipital e hiperintensidad del tracto tegmental central. A. Secuencia T1. B-C-E. Secuencia FLAIR. D. Secuencia T2

\section{Correspondencia:}

Blair Ortiz, Carrera 32 № 7B sur-52, interior 128, Medellín, Colombia.

Teléfonos: (574) 2639856 y (300) 4043964

blairortiz@hotmail.com

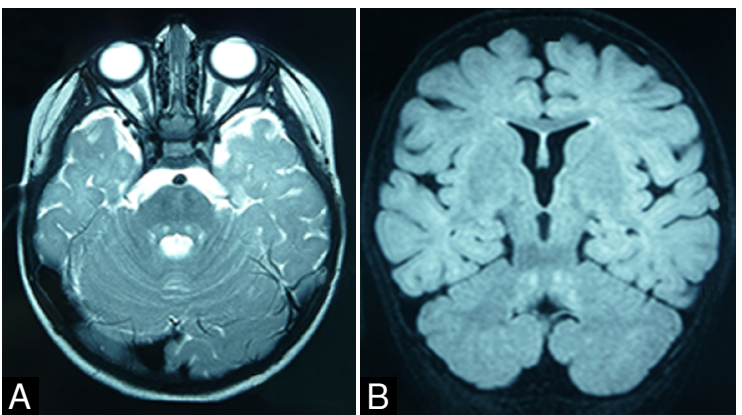

Figura 2. Resonancia magnética cerebral a los 18 meses de vida de la paciente. Se observa solo hiperintensidad del tracto tegmental central y la leucoencefalopatía parieto-occipital ha desaparecido. A. Secuencia T2. B. Secuencia FLAIR

Dada la sospecha de síndrome epiléptico genético, se hizo una secuenciación completa del exoma y se confirmó la mutación del PCDH19 (c.142G>T/ p.Glu48X). Para el control de las convulsiones, fue necesario utilizar hasta cinco medicamentos anticonvulsivos.

A partir de los 18 meses de vida, la niña no volvió a tener convulsiones, pero en la RM cerebral persistía la hiperintensidad del tracto tegmental central, aunque habían desaparecido las lesiones de la sustancia blanca parieto-occipital (figura 2). A los dos años de vida, recibía un solo medicamento anticonvulsivo y presentaba rasgos autistas, en tanto que en el electroencefalograma y la RM cerebral ya no aparecían las anomalías previamente descritas (no se muestran las imágenes).

\section{Discusión}

Las mutaciones en el gen PCDH19 causan epilepsia desde la primera infancia únicamente en el sexo femenino. Hasta hoy, se han encontrado alrededor de 100 mutaciones distintas en este gen, la mayoría de las cuales son esporádicas y, el $50 \%$, sin sentido (nonsense). Después del gen del canal de sodio alfa 1 (SCN1), el PCDH19 es el más relevante en la encefalopatía epiléptica $(7,8)$. El gen $S C N 1 A$ se relaciona con convulsiones febriles simples, epilepsia genética, convulsiones febriles plus y epilepsia mioclónica temprana de la infancia (síndrome de Dravet) $(9,10)$. Hasta un 16 $\%$ de los pacientes que no presentan mutaciones en el SCN1A, las tienen en el PCDH19 $(11,12)$.

En los casos reportados, las crisis ocurren principalmente al final del primer año de vida, con un rango de aparición de 6 a 36 meses de vida $(13,14)$. Es usual encontrar diferentes tipos de crisis, siendo las más comunes las tónicoclónicas, las focales y aquellas en racimo. Con 
menor frecuencia, se pueden presentar otros tipos de crisis, como las mioclónicas, las parciales complejas y las ausencias atípicas $(15,16)$. El estatus epiléptico se ha descrito en $30 \%$ de los pacientes. En el electroencefalograma son usuales las anormalidades frontotemporales. Las crisis se acompañan de fiebre en 54 a $100 \%$ de los casos y en un porcentaje considerable de las pacientes dejan de presentarse al llegar a la adolescencia $(17,18)$.

El fenotipo de las pacientes afectadas por mutaciones en el gen $\mathrm{PCDH} 19$ es heterogéneo. Puede incluir desde la epilepsia con escasas crisis, hasta la encefalopatía epiléptica con deterioro cognitivo y rasgos autistas persistentes. El deterioro cognitivo ocurre en el $70 \%$ de los casos, en el $42 \%$ hay discapacidad intelectual leve, en el $54 \%$, discapacidad intelectual moderada, y en el $4 \%$, discapacidad intelectual grave. Hay rasgos autistas en $28 \%$ de las pacientes $(19,20)$. En este caso, se presentaban muchas de las características del fenotipo descrito por mutación del gen PCDH19: hipotonía, inicio de la epilepsia antes del año, retardo del desarrollo, deterioro del lenguaje y conversión autista, aunque no desarrolló encefalopatía epiléptica. Además, la frecuencia, la duración y la gravedad de las crisis convulsivas, comenzaron a disminuir con la administración de los medicamentos anticonvulsivos, de los cuales solo recibe uno actualmente.

Dado que en la RM cerebral se demostró leucoencefalopatía a los 10 meses de vida, se descartaron leucodistrofia metacromática, adrenoleucodistrofia ligada al cromosoma $X$ (dada su inactivación), enfermedad de la orina con olor a jarabe de arce, hiperglucinemia no cetósica y aciduria orgánica.

Este es el primer reporte de caso de una paciente afectada por la mutación del gen PCDH19 asociada con leucoencefalopatía y tractopatía posterior reversible. Mediante las RM cerebrales se evidenció que las lesiones involucionaron progresivamente $\mathrm{y}$, a los 24 meses de vida, las imágenes eran normales.

\section{Conclusión}

Este reporte de caso aporta información adicional para estudiar la mutación del gen PCDH19 en pacientes de sexo femenino con leucoencefalopatía de causa no metabólica. En este sentido, sería recomendable ofrecer la posibilidad de secuenciación del gen $\mathrm{PCDH} 19$ a las pacientes con epilepsia, retardo del desarrollo y lesión de los cuadrantes posteriores, una vez se descarte un error innato del metabolismo.

\section{Agradecimientos}

A la paciente y a su familia, por facilitar todo el historial clínico y las ayudas diagnósticas a los investigadores, así como por su autorización para esta publicación.

\section{Conflicto de intereses}

Los autores declaran no tener conflicto de intereses.

\section{Financiación}

Los autores no recibieron recursos económicos.

\section{Referencias}

1. Hirabayashi T, Yagi T. Protocadherins in neurological diseases. Adv Neurobiol. 2014;8:293-314.

2. Angst BD, Marcozzi C MA. The cadherin superfamily: Diversity in form and function. J Cell Sci. 2001;114:629-41.

3. Biswas S, Emond MR, Jontes JD. Protocadherin-19 and $\mathrm{N}$-cadherin interact to control cell movements during anterior neurulation. J Cell Biol. 2010;191:1029-41. https:// doi.org/10.1083/jcb.201007008

4. Dibbens LM, Tarpey PS, Hynes K, Bayly MA, Scheffer IE, Smith R, et al. X-linked protocadherin 19 mutations cause female-limited epilepsy and cognitive impairment. Nat Genet. 2008;40:776-81. https://doi.org/10.1038/ng.149

5. Liu $\mathbf{Q}$, Sunil Bhattarai, Wang $\mathbf{N}$, Sochacka-Marlowe A. Differential expression of protocadherin-19, protocadherin-17 and cadherin-6 in adult zebrafish brain. J Comp Neurol. 2016;8:583-92. https://doi.org/10.1002/cne. 23746

6. Emond MR, Biswas S, Jontes JD. Protocadherin-19 is essential for early steps in brain morphogenesis. Dev Biol. 2009;334:72-83. https://doi.org/10.1016/j.ydbio.2009.07.008

7. Cooper SR, Jontes JD, Sotomayor M. Structural determinants of adhesion by protocadherin-19 and implications for its role in epilepsy. Elife. 2016;5:1-22. https://doi.org/10.7554/eLife.18529

8. Depienne C, LeGuern E. PCDH19-related infantile epileptic encephalopathy: An unusual X-linked inheritance disorder. Hum Mutat. 2012;33:627-34. https://doi.org/10.1002/ humu.22029

9. Fejerman $\mathbf{N}$. Etiologías genéticas asociadas con epilepsias graves del lactante. Arch Argent Pediatr. 2012;110:421-9. https://doi.org/10.1590/S0325-00752012000500010

10. van Harssel JJ, Weckhuysen $\mathbf{S}$, van Kempen $\mathbf{M J}$, Hardies K, Verbeek NE, De Kovel CG, et al. Clinical and genetic aspects of $P C D H 19$-related epilepsy syndromes and the possible role of $P C D H 19$ mutations in males with autism spectrum disorders. Neurogenetics. 2013;14:23-34. https://doi.org/10.1007/s10048-013-0353-1

11. Kwong AK, Fung CW, Chan SY, Wong VC. Identification of SCN1A and PCDH19 mutations in Chinese children with 
Dravet syndrome. PLoS One. 2012;7:e41802. https://doi. org/10.1371/journal.pone.0041802

12. Baulac S, Gourfinkel-An I, Nabbout R, Huberfeld G, Serratosa J, Leguern E, et al. Fever, genes, and epilepsy. Lancet Neurol. 2004;3:421-39. https://doi.org/10.1016/S14744422(04)00808-7

13. Specchio N, Fusco L, Vigevano F. Acute-onset epilepsy triggered by fever mimicking FIRES (febrile infectionrelated epilepsy syndrome): The role of protocadherin 19 (PCDH19) gene mutation. Epilepsia. 2011;52:172-5. https:// doi.org/10.1111/j.1528-1167.2011.03193.x

14. Sharma S, Prasad AN. Genetic testing of epileptic encephalopathies of infancy: An approach. Can J Neurol Sci. 2013;40:10-6. https://doi.org/10.1017/S0317167100012889

15. Liu A, Xu X, Yang X, Jiang Y, Yang Z, Liu X, et al. The clinical spectrum of female epilepsy patients with $\mathrm{PCDH} 19$ mutations in a Chinese population. Clin Genet. 2017;91:54 62. https://doi.org/10.1111/cge.12846

16. Marini C, Mei D, Parmeggiani L, Norci V, Calado E, Ferrari A, et al. Protocadherin 19 mutations in girls with infantile-onset epilepsy. Neurology. 2010;75:646-53. https:// doi.org/10.1212/WNL.0b013e3181ed9e67

17. Marini C, Darra F, Specchio N, Mei D, Terracciano A, Parmeggiani L, et al. Focal seizures with affective symptoms are a major feature of $P C D H 19$ gene-related epilepsy. Epilepsia. 2012;53:2111-9. https://doi.org/10.1111/ j.1528-1167.2012.03649.x

18. Antelmi E, Mastrangelo M, Bisulli F, Spaccini L, Stipa C, Mostacci B, et al. Semiological study of ictal affective behaviour in epilepsy and mental retardation limited to females (EFMR). Epileptic Disord. 2012;14:204-309. https:// doi.org/10.1684/epd.2012.0526

19. Higurashi N, Nakamura M, Sugai M, Ohfu M, Sakauchi M, Sugawara Y, et al. PCDH19-related female-limited epilepsy: Further details regarding early clinical features and therapeutic efficacy. Epilepsy Res. 2013;106:191-9. https://doi.org/10.1016/j.eplepsyres.2013.04.005

20. Gagliardi M, Annesi G, Sesta M, Tarantino P, Conti P, Labate A, et al. PCDH19 mutations in female patients from Southern Italy. Seizure. 2015;24:118-20. https://doi. org/10.1016/j.seizure.2014.08.010 